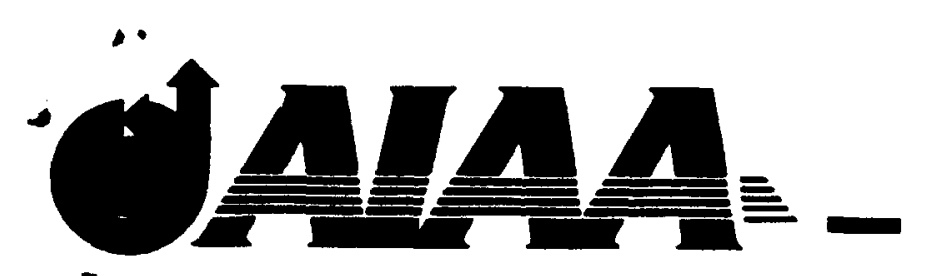

AD-A268 794

AIAA-93-0211

Interferometric Investigations of

Compressible Dynamic Stall Over a

Transiently Pitching Airfoil

M.S.Chandrasekhara, Naval Postgraduate

School, Monterey, CA;

L.W.Carr, U.S.Army ATCOM,

NASA Ames Research Center,

Moffett Field, CA; and

M.C.Wilder, MCAT Institute, San Jose, CA
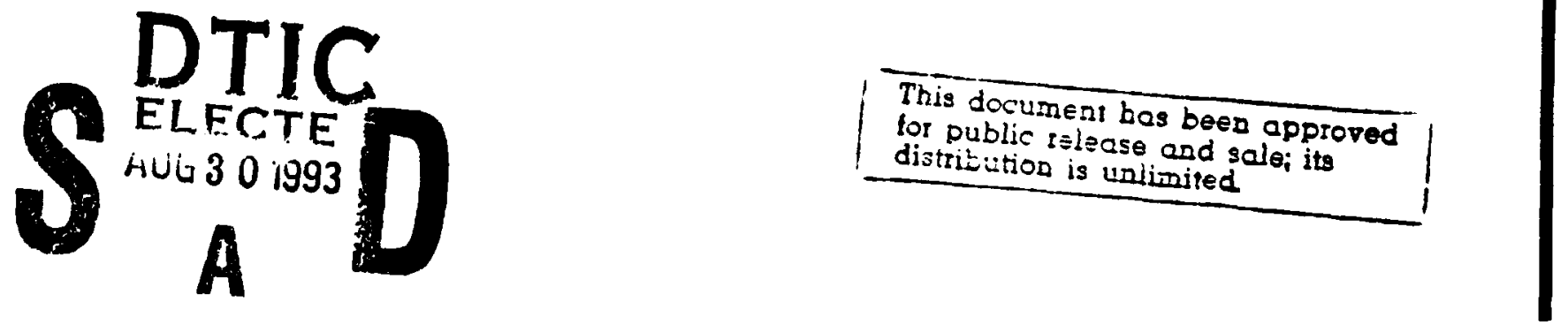

$93824 \quad 219$

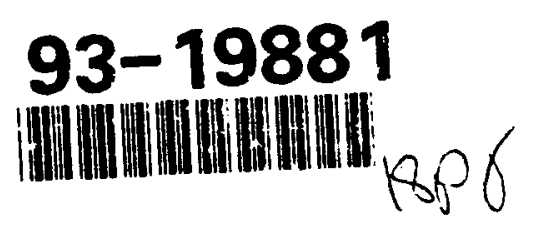

31st Aerospace Sciences

Meeting \& Exhibit

January 11-14, 1993 / Reno, NV 


\title{
Interferometric Investigations of Compressible Dynamic Stall Over a Transiently Pitching Airfoil Accesion For
}

\author{
M.S. Chandrasekhara ${ }^{1}$ \\ Navy-NASA Joint Institute of Aeronautics \\ Department of Aeronautics and Astronautics \\ Naval Postgraduate School, Monterey, CA 93943 \\ L.W. Carr ${ }^{2}$ \\ U.S.Army ATCOM and Fluid Mechanics Laboratory Branch \\ NASA Ames Research Center, Moffett Field, CA 94035-1000 \\ and \\ M.C. Wilder ${ }^{3}$ \\ Navy-NASA Joint Institute of Aeronautics and \\ MCAT Institute, San Jose, CA
}

\begin{abstract}
The dynamic stall flow field over a NACA 0012 airfoil pitching transiently from $0-60^{\circ}$ at a constant rate under compressible flow conditions has been studied using the real-time technique of point diffraction interferometry. This investigation using nonintrusive diagnostics provides a quantitative description of the overall flow field, including the finer details of dynamic stall vortex formation, growth and the concomitant changes in the pressure distribution. Analysis of several hundred interferograms obtained for a range of flow conditions shows that the peak leading edge suction pressure coefficient at stall is nearly constant for a given free stream Mach number at all nondimensional pitch rates. Also, this value is below that seen in steady flow at static stall for the same Mach number, indicating that dynamic effects significantly affect the separation behavior. Further, for a given Mach number, the dynamic stall vortex seems to form rapidly at nearly the same angle of attack for all pitch rates studied. As the vortex is shed, it induces an anti-clockwise trailing edge vortex, which grows in a manner similar to that of a starting vortex. The measured peak suction pressure coefficient drops as the free stream Mach number increases. For free stream Mach numbers above 0.4, small multiple shocks appear near the leading edge
\end{abstract}

${ }^{1}$ Associate Director and Research Associate Professor, Assoc. Fellow AIAA,

Mailing Address: M.S. 260-1, NASA Ames Research Center, Moffett Field, CA 940351000

${ }^{2}$ Research Scientist and Group Leader, Unsteady Viscous Flows, Aeroflightdynamics Directorate, Member AIAA

\section{${ }^{3}$ Research Scientist}

This paper is declared a work of the U.S. Government and is not subject to copyright protection in the United States.

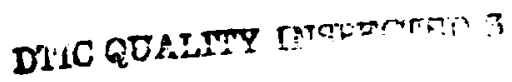

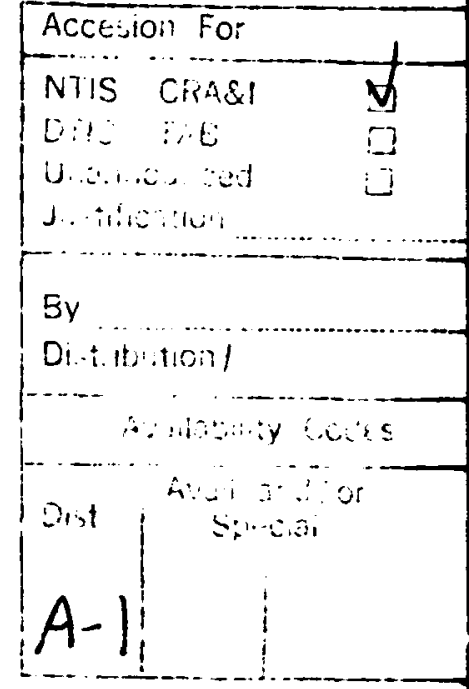

above the shear layer. The unsteady flow peak suction pressure coefficient at any angle has been experimentally demonstrated to lag that of the steady flow at the same angle of attack.

\section{Nomenclature}

$\begin{array}{ll}C_{p} & \text { pressure coefficient } \\ \text { c } & \text { airfoil chord } \\ \mathbf{M} & \text { free stream Mach number } \\ \mathrm{Re} & \text { Reynolds number based on } c \text { and } U_{\infty} \\ U_{\infty} & \text { free stream velocity } \\ \mathbf{x}, \mathrm{y} & \text { chordwise and vertical distance } \\ \alpha & \text { angie of attack } \\ \dot{\alpha} & \text { pitch rate in } \mathrm{deg} / \mathrm{sec} \\ \alpha^{+} & \text {nondimensional pitch rate, } \frac{\dot{\alpha} c}{U_{\infty}} \\ \gamma & \text { ratio of specific heats } \\ \epsilon & \text { fringe number } \\ \rho & \text { density } \\ \rho_{r} & \text { density at reference(atmospheric) conditions }\end{array}$

\section{Introduction}

The utilization of dynamic stall as a method for increasing the maneuverability and agility of aircraft has received significant attention during the past few years. Several researchers ${ }^{1-6}$ have studied the flow over pitching airfoils using flow visualization and unsteady pressure measurements and provided valuable information on the dynarnic stall phenomenon. However, all these studies are at low speeds. Lorber and Carta ${ }^{7}$ have obtained measurements under compressibility conditions which showed that at the higher Mach number, the flow could not develop the suction levels observed under incompressible conditions, and noted that this effectively limited the stall delay that could be achieved. The computational studies of $\mathrm{Visbal}^{8}$ lend some support to this result. Since compressibility effects have been shown ${ }^{9,10}$ to change the way that dynan:ir stall deveiups, a ietter understanding of these effects has been of interest in the development of supermaneuverable aircraft and highly agile 
encoder, whose output was input to the digital I/O board of a microVAX II Work Station and timed with its internal clock. Figure 2 shows an example of the actual rates obtained, including the variation of the angle of attack during the various parts of the pitchup motion in an experiment. As can be seen, the airfoil goes through its static stall angle with a linear rate of change of angle of attack. For the highest rate, the motion is completed in $18 \mathrm{~ms}$, beyond which the system is settling down (at the highest angle). All the tests were limited to the linear range.

The CDSF is equipped with a wide range of nonintrusive optical flow diagnostic instrumentation such as stroboscopic schlieren, laser Doppler velocimetry, holographic interferometry and point diffraction interferometry systems. The present paper will present results obtained using the point diffraction interferometry system.

\subsection{The Point Diffraction Interferometry Technique}

The point diffraction interferometry technique used in this study utilizes the ability of a point discontinuity (in the form of a pin-hole) located at the image of a point source to diffract a portion of the incident light into a spherical reference wave front. In the present application, the primary optics of an existing schlieren system were used (see Reference 13 for details), with a pulsed Nd:YAG laser replacing the conventional spark as the light source, and a specially created point diffractor replacing the usual knife edge. The laser light was expanded through a microscope objective to fill the schlieren mirror, transmitted through the test section, and refocused by another schlieren mirror. An exposed photographic plate used to create the point-diffraction spot was placed at the focus of this second mirror, and the laser was pulsed with enough energy to burn a hole, or spot, in the emulsion located at the focal plane of the second mirror. The spot was created in situ by passing light through the test section at a no-flow condition. The spot was precisely tailored to the application under investigation, automatically correcting for nonuniformities in the light source or optics. With the flow turned on, the laser was triggered externally at the desired angles of attack and the real-time interference fringes were recorded on Polaroid film(ASA 3000), which were available for immediate viewing. More details about the PDI technique can be found in References 13 and 14.

\subsection{Interferogram Image Processing}

The quantitative nature of the interferograms enabled computation of the pressure distribution over the airfoil when the flow was attached. The interferograms were processed in a semi-automatic mode using a specially developed software package. The program read a digitized (256 gray level) interferogram on an IRIS Work Station and overlayed an airfoil using the triangular registration markers seen in the images. The intersection of the fringes with the airfoil upper and lower surfaces (or the local boundary layer edge, when detectable) were interactively picked by the user. Since each fringe is a line of constant density, the corresponding pressure at the boundary layer edge could be derived using isentropic flow relations. This pressure was then used as the surface pressure, under the boundary layer assumptions. The density along any fringe could be calculated from the Gladstone - Dale equation ${ }^{15}$, which for the present wind tunnel and laser simplifies to

$$
\rho-\rho_{r}=0.009421 \epsilon
$$

where $\epsilon$ the fringe number is $0, \pm 1, \pm 2, \ldots$ for the bright fringes and $\pm \frac{1}{2}, \pm \frac{3}{2}, \pm \frac{5}{2}, \ldots$ for the dark fringes. Fringes from the free stream to the stagnation point have positive values. The corresponding $C_{p}$ distributions were then computed from the relation

$$
C_{p}=\frac{\left[\left(\frac{\rho}{\rho_{r}}\right)^{\gamma}-1\right]}{\left[\frac{\gamma}{2} M^{2}\right]}
$$

In the end, the package provided an output data file containing the various physical variables, in a format suitable for plotting. Typical processing time was about 3 - 5 minutes per image.

In cases where the fringe density was high or the fringes were fuzzy, the user could go into the 'off-body' mode and pick fringes along a line parallel to and away from the airfoil surface where the fringes are farther apart. For this purpose, an option to superpose two larger airfoils over the image on the screen was provided. The fringe intersections on the larger airfoils were then projected to the airfoil surface suitably. At angles of attack near the dynamic stall angle, the fringes near the leading edge region were very dense reflecting the large local density gradients. Further, in this region, optical noise introduced by the shadowgraph effect generally lowered the contrast, making it a location where the off-body mode needed to be invoked.

In the present study the entropy change in the vortical flow was ignored (for lack of a better method). Interferograms with shocks have not been processed because of this limitation.

\subsection{Experimental Conditions}

The Mach number of the experiment ranged from $0.2-0.45$. The corresponding nondimensional pitch rate varied from 0.020 to 0.045 , depending on the Mach number. The actual maximum rate, as stated, was limited to 3600 degrees/sec. The images were obtained at a resolution of 0.5 degrees, with some exceptions when the results indicated need for a better resolution.

The nondimensional pitch rates reported are based on the total time for pitching from $0^{\circ}-57^{\circ}$. However, the hydraulic control system of the pitching drive caused the airfoil to pitch $5-8 \%$ faster in the $0^{\circ}-10^{\circ}$ range and sometimes in the $0^{\circ}-30^{\circ}$ range. This difference is not believed to significantly affect the global results of the study.

The experiments were conducted in two phases. In the first phase, the full flow field interferograms were obtained for a range of conditions: phase II focused on the leading edge flow details only. 


\section{Results and Discussion}

The interferogram images will be first discussed qualitatively as flow visualization images. The quantitative pressure distributions derived from the images will be presented in the second part of the section. Whereas much of the paper is devoted to the leading edge flow, when appropriate, the full flow field is also discussed.

\subsection{Discussion of Interferogram Images}

\section{A. Separation Bubble and Dynamic Stall}

Fig. 3 is a point diffraction interferogram of the flow at $M=0.3, \alpha=12^{\circ}$ and $\alpha^{+}=0.03$. This image reveals some important features of the flow. The dark closed fringe on the lower surface slightly aft of the leading edge is the stagnation point fringe. The suction pressure developed by the airfoil causes the local flow to accelerate, resulting in strong density changes, which is seen in the figure as a concentration of fringes near the leading edge on the upper surface. The close spacing of the fringes also means that the flow gradients are very high. In fact, 21 dark fringes are present in about 1 millimeter $(x / c \approx 0.1)$ in this image, indicating that the local maximum Mach number is 0.71 and the local pressure coefficient is -3.75 at $x / c=0.01$. Downstream of this point, a steep adverse pressure gradient region develops(see Sec. 3.2.C) and classical laminar flow separation occurs. The separated shear layer reattaches after it transitions into a turbulent layer, forming a bubble. In the figure, the fringes in the bubble appear as lines emanating from the leading edge which turn abruptly towards the upper surface at $x / c \approx 0.05$ and turn sharply again as the local boundary layer is encountered. Inside the bubble, the fringes run parallel to the surface locally, representing the pressure plateau normally associated with laminar separation bubbles. The accompanying pressure distribution (which will be discussed in Sec. 3.2.C) obtained by processing the image using the fringe analysis software shows the suction peak, the drop in suction due to the adverse pressure following it and the laminar separation bubble, which is indicated by the plateau in the distribution. In the interferograms to be discussed, all of the features upstream of the bubble are found. As dynamic stall occurs, differences evolve which will be pointed out.

\section{B. Leading Edge Supersonic Flow}

Chàndrasekhara et al ${ }^{16}$ found that the airfoil leading edge flow can become supersonic leading to formation of multiple shocks. Fig. 4 provides quantitative documentation of this. Depending upon the angle of attack, a shock, or multiple shocks form in the flow. Fig. 4 shows a PDI image for $M=0.45$, at $\alpha=12.6^{\circ}$ and $\alpha^{+}=0.0313$. Fringe counting shows that the local Mach numher aheal if the shork is greater than 1.0 and at the foot of the shock, it is about 1.2. Although the flow is only weakly supersonic, the shock causes the leading edge laminar boundary layer to separate ${ }^{16}$. This separated free shear layer develops a waviress, which causes the flow downstream of the shock to go through a series of ac- celerations and decelerations. As the flow negotiates the crests and valleys of this wavy shear layer, expansion waves and compression waves develop, causing a series of shocks. The last shock in the series appears to be the strongest and the flow becomes subsonic downstream. The occurrence of multiple shocks is repeatable, and the shocks were found to be present over an angle of attack range of about one degree.

\section{Flow Description at $\mathrm{M}=0.2, \alpha^{+}=0.03$}

Fig. 5 presents a montage of interferograms for $M=0.2$ at a nondimensional pitch rate of 0.03 for $12^{\circ} \leq \alpha \leq 24.75^{\circ}$. A separation bubble is present for $\alpha=12^{\circ}$; analysis of the interferograms showed that the bubble first appeared at $\alpha=7^{\circ}$ (as opposed to about 6 degrees in steady flow at $M=0.2$ ). At $\alpha=15^{\circ}$, the fringe near the trailing edge indicates a mild local flow separation as this fringe first moves into the wake and turns sharply back towards the trailing edge. Also, the leading edge bubble starts to open-up and the first imprint of the dynamic stall vortex becomes distinct at $\alpha=17^{\circ}$. As it grows, the enveloping shear layer moves downstream and the airfoil boundary layer thickens. The growth of the vortex continues while it convects over the airfoil during the continuous ramping motion. For example, at $\alpha=18^{\circ}$, the center of the vortex is at $\mathrm{x} / \mathrm{c}=0.1$, but the downstream edge of the surrounding shear layer is at $x / c=0.3$. The number of fringes is seen to increase till $\alpha=18^{\circ}$ demonstrating that the low pressure region continues to grow. By $\alpha=20^{\circ}$, the leading edge flow has separated and the fluid aft of the shear layer enclosing the vortex has been convected past the trailing edge and thus, no fringes can be seen in this region. Once the vortex grows and begins to convect, the innermost fringes become circular (for example at $\alpha=21^{\circ}$ and $22^{\circ}$ ), whereas the outer fringes still end on the surface. The number of fringes inside the vortex is now larger than that at the leading edge. This implies that the pressure in the vortex core is lower than the peak suction over the airfoil. Surface pressure measurements, however, cannot reveal this. The vortex is eventually shed by $\alpha=24.75^{\circ}$, and the flow reaches the deep stall state. A counter clockwise trailing edge vortex also forms at this angle of attack. For this test condition, the vortex remains over the surface for a large angle of attack range of 12 degrees. Similar studies on an oscillating airfoil $\left(\alpha=10^{\circ}+10^{\circ} \sin \omega t\right)$ at $M=0.2$ showed that deep stall occurred at $\alpha=18.1^{\circ}$. The presence of the dynamic stall vortex till $\alpha=24.75^{\circ}$ elicits the fact that motion history plays a key role in the dynamic stall process. In this case, the transiently pitching airfoil is found to be better in sustaining the dynamic lift generated.

D. Flow Sequence at $\mathrm{M}=0.45, \alpha^{+}=0.02$.

Fig. 6 shows a similar set of interferograms for $M$ $=0.45$ at a pitch iate of 0.02 . Ai this Mach number, compressibility effects dominate ${ }^{16}$. The large number of fringes seen at low angles of attack are due to the larger density changes in the flow at this higher Mach number. The innermost closed fringe intersecting the lower surface near the leading edge encloses the stagnation point. There are 58 fringes (corresponding to 
a local mach number of $\approx 1.2$ ) around the leading edge in the first $1.5 \%$ of the airfoil chord at $\alpha=9^{\circ}$. A separation bubble forms in this case also and is seen clearly at $\alpha=7^{\circ}$. In steady flow at this Mach number, the bubble was first found for $\alpha=5^{\circ}$, but a delay was observed in its first appearance in the unsteady case. At $\alpha=9^{\circ}$, the first signs of the dynamic stall vortex/vortical structure are seen as a thin shear layer between the bubble and the airfoil upper surface near the leading edge region(see also Fig. 7). Multiple shocks can be seen above the airfoil shear layer and the flow is similar to that discussed in Fig. 4. The shocks remain on the surface till $\alpha=11^{\circ}$, even after the dynamic stall process is well under way. It is not yet clear whether the first shock induced boundary layer separation caused the dynamic stall vortex to form.

The vortex grows as the shear layer enveloping it reattaches further down the airfoil towards the trailing edge with increase in angle of attack. As in the low Mach number case, the fluid aft of this point has been swept away into the wake. This is a region of nearly stagnant fluid. No circular fringes are found in the vortex; instead, only half circular fringes are seen. At $\alpha=14.5^{\circ}$, the edge of the shear layer has reached the trailing edge, and deep stall occurs at $\alpha=15.5^{\circ}$. The entire sequence lasts only $6-7 \mathrm{de}-$ grees in angle of attack, which is considerably smaller than the $12^{\circ}$ range seen for $M=0.2$. Although the lower Mach number result discussed was obtained at a nondimensional pitch rate which was $50 \%$ higher, the range of angle of attack over which dynamic lift is sustained is nearly twice that seen at $M=0.45$. This confirms the result obtained from the earlier schlieren studies $^{16}$ that compressibility promotes stall. These results agree with those reported in Ref. 17 for the oscillating airfoil. However, it should be noted that the two different motion histories will force differences in the details of separation. Dynamic stall of oscillating airfoils is influenced by hysteresis which affects all aspects of the flow. The transiently pitching airfoil flow is free of this parameter. In addition, since the airfoil continues to pitch to $60^{\circ}$, which is well beyond the static stall angle, the development of the post-stall flow and the interactions at the trailing edge can be studied (discussed in Sec. F), which is of importance to the supermaneuverability problem. This information is also of value in comparing computed results against experiments.

The differences in the vortex size and structure also imply that the overall (global) pressure field in the incompressible and compressible flow cases are quite different. The sustained presence of a low pressure region over the airfoil upper surface is evidence that even in the compressible case, there is increased lift generated during the dynamic stall process.

\section{E. Leading Edge Flow Stall Vortex Formation}

Carr et al ${ }^{17}$ found that for an oscillating airfoil, the dynamic stall vortex formed just as the separation bubble burst. It has now been found to be true for the transiently pitching airfoil also. Fig. 7 presents some interferograms that demonstrate this result for $\mathrm{M}=$ 0.3 and $\alpha^{+}=0.03$. At $\alpha=15^{\circ}$, the fringes enclosing the bubble at a lower angle of attack (not shown) begin to 'open-up' and immediately appear above the airfoil, fringes normal to the surface appear near the downstream end of the bubble. This can be seen more clearly at $\alpha=15.5^{\circ}$. By $\alpha=15.75^{\circ}$, these fringes extend to about $1.5 \%$ of chord above the airfoil and nearly to $0.2 \mathrm{c}$ along it. The inflexion in the fringes very close to the airfoil surface suggests that there is a very slight reverse flow. In contrast, the outer fringes (outside of the bubble) proceed only in one direction, towards the trailing edge. The region of reverse flow is less than $0.005 \mathrm{c}$ and thus, it is extremely difficult to detect with experimental techniques other than surface mounted gages. By $\alpha=16.5^{\circ}$, the vortex has fully developed and convected to $0.2 \mathrm{c}$. This rapid succession of events in a very small angle of attack range is typical of the evolution of dynamic stall at compressible Mach numbers. However, the interaction between shocks and the boundary layer, along with the events of bubble bursting and vortex formation are too complicated to be resolved from the images processed so far.

The primary issue that needs to be determined is the origin of the dynamic stall vortex. Whether it originates independently of the separation bubble and simply pushes the back end of the bubble, or the increasing angle of attack makes it no longer possible for the bubble to remain close to the leading edge and moves the reattachment point rearward allowing the leading edge vorticity to coalesce, is yet to be found. This is an intriguing issue, especially because the leading edge flow has already separated, causing the bubble, at a very low angle of attack (much lower than the static stall angle). A plausible description is that the recirculation region in the bubble becomes stronger with increase of angle of attack, eventually forcing a breakdown of the leading edge flow, triggering the vortex formation. However, a much more careful analysis is needed for clarifying the issues.

The formation of a separation bubble indicates that the airfoil leading edge boundary layer transition plays a critical role in dynamic stall occurrence. If the boundary layer is modified to transition before the adverse pressure gradient is encountered, it is to be expected that dynamic stall and the associated events occur in a very different manner. This includes stall onset, the details of the vortex formation and the duration of dynamic lift in the pitching cycle. This is a further aspect that merits a more detailed study.

\section{F. Trailing Edge Flow Beyond Deep Stall}

The flow continues to show interesting features even after deep stall has occurred and the dynamic stall vortex is shed. In Fig. 8 , the complete flow field is shown for just one experimental flow condition $\left(\mathrm{M}=0.45\right.$ and $\left.\alpha^{+}=0.025\right)$. For this case, the dynamic stall angle of attack (when the dynamic stall vortex leaves the airfoil) is 17 degrees; however, the airfoil continues to $\therefore \mathrm{Ch}$ at a constant iate liii $\alpha=60^{\circ}$. An immediate consequence of vortex shedding under these conditions is an increase in suction pressure at the trailing edge on the lower surface of the airfoil. The trailing edge suction gradually increases during the dynamic stall process as the airfoil pitches from $C_{p} \approx 0.0$ at $\alpha=0^{\circ}$ to $C_{p}=-0.504$ at $\alpha=17^{\circ}$. However, at $\alpha=17.5^{\circ}$. the suction pressure coefficient jumps to -1.04 . Also, a counter clock wise 
vortex begins to form in the near-wake, at $\alpha=18^{\circ}$. The birth/growth of this vortex pushes the separating shear layer from the leading edge outward. At $\alpha=20^{\circ}$, this vortex is shed and by $\alpha=30^{\circ}$. only the separated flow can be seen over the upper surface. The same number of fringes $(26$, corresponding to $C_{p}=-1.08$ ), measured from the stagnation point to this point on the upper and lower surface shear layers seems to indicate that the pressure over the upper surface is nearly constant. However, since the field of view is limited in the facility, the role of the wake and the interaction of the two shear layers cannot be determined to ascertain this fully. As the pitch up continues, shear layer instabilities develop, which roll up into vortices, which appear at $\alpha=50^{\circ}$ or $55^{\circ}$. A large trailing edge vortex appears from the lower surface at $\alpha=50^{\circ}$, which rolls up towards the leading edge shear layer. The peak suction increases slightly with angle of attack, even though the airfoil is in the deep stall state. The stagnation point moves to 0.25 chord point at $\alpha=55^{\circ}$. Similar features were found for other Mach numbers as well. However, this picture is significantly different from the low Reynolds number and low Mach number results of Walker et $\mathrm{al}^{5}$, who observed two large vortices co-existing on the airfoil suction surface as dynamic stall progressed. This led to a much different airfoil surface pressure distribution than found here. For all the cases studied here, the trailing edge vortex was induced as the dynamic stall vortex was shed. At times the leading edge separated shear layer produced a large vortex, resulting a double vortex pattern. But, it then had the appearance of a vortex street.

\subsection{Quantitative Pressure Field}

One of the main advantages of PDI is its truly nonintrusive way of yielding the pressure field. In a rapidly changing flow such as the ramping airfoil motion under consideration, it requires several realizations of the flow - i.e. several pitch up motions - to obtain a surface pressure distribution by standard techniques. The PDI technique not only provides the surface pressure map, but also the global pressure field instantaneously in one picture, without any history effects present in it. Since a large number of interferograms were obtained at close intervals in angle of attack, a finer set of instantaneous pressure data is in hand now. The data to be reported are the first global mapping pressure coefficients that have been obtained for a transiently pitching airfoil under compressibility conditions using PDI.

\section{A. Effect of Mach Number on Peak Suction Pressure}

Fig. 9 shows the peak suction pressure coefficient obtained over the airfoil at different Mach numbers for different pitch rates. The absolute value of $C_{P}$ in steady flow is lower than in unsteady flows for all Mach numbers tested. Of significance is the relative independence of the peak suction pressure coefficient of the pitch rate for all Mach numbers. Also, the peak suction decreases dramatically with increasing Mach number. For the lowest Mach number of 0.2 , the value of $C_{p}=-7.1$ corresponds to a local Mach number of 0.58 , indicating that compressibility conditions play a role in the process. At $\mathbf{M}=0.45$. the sonic limit is $C_{p}=-2.78$ and with the suction peak at $C_{p}=$ -3.6 , the local flow is supersonic and thus is strongly compressible. It is possible that the formation of the bubble and the strong local compressible conditions have both affected the viscous/inviscid interactions in the flow and thus, limited the suction pressure from increasing as the pitch rate is increased.

\section{B. Effect of Pitch Rate}

In Fig. 10, the development of the leading edge pressure distribution over the first $5 \%$ of airfoil chord is compared for $\mathbf{M}=0.3$ for steady flow; $\alpha^{+}=0.035$ and $\alpha^{+}=0.04$ at $\alpha=10$ degrees. It is clear that steady flow develops a stronger suction peak than the unsteady flow cases, with a maximum $C_{p}$ of -3.6 ; the suction peak is located at $\mathrm{x} / \mathrm{c}=0.012$. The adverse pressure gradient is slower to develop at the higher pitch rates; in fact, the suction level has reached only a value of $C_{p}=-2.95$ at 10 degrees angle of attack. The pressure distribution for the unsteady cases is flatter than in the steady flow case as observed from the interferograms. Even though all three cases show a separation bubble, as observed on the interferograms, th effect of increasing pitch rate is clearly to delay the flow development over the leading edge, which consequently leads to delay in dynamic stall occurrence.

Fig. 11 shows the maximum suction pressure coefficient at different angles of attack for $\mathbf{M}=0.4$. In it, steady flow, and unsteady flows at $\alpha^{+}=0.02$ and $\alpha^{+}=0.035$ are compared. The steady flow stalls at $\alpha=10.8^{\circ}$; at higher angles the leading edge suction falls rapidly. At $\alpha^{+}=0.02$ the effect of decreased adverse pressure gradient as seen in Fig. 8 is seen at all angles of attack; the suction levels for $\alpha^{+}=0.035$ lag those of even $\alpha^{+}=0.02$. The rounding of the curves at the top corresponds to the situation when the dynamic stall vortex is in the formative stages. During this stage $\left(\alpha=12^{\circ}-13^{\circ}\right.$ for $\alpha^{+}=0.02$ and $\alpha=13^{\circ}-13.5^{\circ}$ for $\alpha^{+}=0.035$ ) the airfoil suction is the maximum. Once the vortex begins to convect, the leading edge flow slows down and the suction is steadily lost, even though the angle of attack is still increasing. Even though there is experimental scatter, it is also clear that dynamically pitching airfoils can withstand larger suction peaks than steady airfoils prior to stall and withstand higher flow gradients, before stall occurs. It is also of value to note that the loss of suction peak in the dynamic case does not mean loss of lift, but only the initiation of the dynamic stall vortex.

Fig. 12 shows the pressure distribution at $\mathbf{M}=$ 0.3 at $\alpha=10^{\circ}$ in steady flow and $\alpha=11^{\circ}$ in unsteady flow at $\alpha^{+}=0.035$. The nearly identical pressure distributions suggest that unsteadiness essentially has introduced a one degree lag in the effective angle of attack, including the formation of the separation bubble (see next section), supporting the conclusions drawn from Fig. 10 and Fig. 11. As much as two degrees of decrease in the effective angle of attack was observed for oscillating airfoils by Carr et $\mathrm{al}^{17}$. However, as can be expected, the amount of this benefit is determined by the experimental conditions, especially the pitch rate and Mach number. 


\section{Airfoil Pressure Distributions}

The airfoil pressure distributions presented in Fig. 13 were obtained for different Mach numbers and pitch rates using the method described earlier. Only one typical case is discussed below for $M=0.3$, $\alpha^{+}=0.035$ at different angles of attack as seen in Fig. 13. For this case, Fig. 13a shows that at $\alpha=5^{\circ}$, the pressure distribution is smooth, with the suction peak of $C_{p}=-1.69$ at $\mathrm{x} / \mathrm{c}=0.025$. As the airfoil pitches to 7 degrees, $C_{p}$ increases to -2.21 . This is accompanied by the upstream movement of the suction peak to $x / c=0.015$. Along with this, the stagnation point moves from $\mathrm{x} / \mathrm{c}=0.01$ to $\mathrm{x} / \mathrm{c}=0.05$. As the airfoil pitches to higher angles of attack, further movement of the stagnation point is difficult to detect, until larger angles are reached. At $\alpha=8^{\circ}$, a separation bubble forms. The pressure plateau following the steep drop in the pressure distribution immediately after the suction peak at $\alpha=9^{\circ}$ confirms this. The bubble extends to $x / c \approx 0.05$. The suction level and the adverse pressure gradient both continue to increase as the airfoil angle of attack is increased. However, Fig. 13b shows that the pressure distributions at $\alpha=13^{\circ}$ and $\alpha=14^{\circ}$ are only incrementally different from that at $\alpha=12^{\circ}$, indicating that the lift and thus, the circulation over the airfoil have not changed significantly in this range of pitching. It is also the range when the dynamic stall vortex is initiated as determined by visual analysis of the interferogram images. The suction peak, however, increases with angle of attack and reaches its maximum value of -5.8 when the first imprint of dynamic stall is clearly discernible in the images. At this instant, it appears that the vortex becomes fully organized and then it begins to convect. The movement of the vortex results in a slight drop of airfoil suction. However, the pressure distribution changes to the nearly flat-top shape which extends to about $x / c=0.3$. This increasing region of low pressure over the upper surface is the cause of the vortical lift seen in the flow. Further pitching of the airfoil continues to decrease the suction levels, while spreading its extent more over the airfoil, until it is shed past the trailing edge.

\section{Concluding Remarks}

A nonintrusive study of the compressibility effects on dynamic stall of a transiently pitching airfoil has been conducted. The study shows the following:

1. Dynamic stall occurs rapidly as the separation bubble bursts. This points to the need for better experimental and computational modelling of the boundary layer transition process.

2. Multiple shocks form over the airfoil for a free stream Mach number of 0.4 and higher. The role of the shocks in altering the dynamic stall process needs a careful study.

3. Compressibility lowers the angle of attack at which stall occurs, primarily due to unfavorable interaction with the airfoil boundary layer.

4. The duration of dynamic lift lasts over a considerably smaller angle of attack range when compressibility effects are strong.

5. There is marked differences in the vortex structure between the compressible and incompressible cases.

6. Increasing Mach number decreases the maximum $C_{p}$ developed by the airfoil. However, for a given Mach number it is relatively independent of the pitch rate.

7. Interferogram images confirm the low pressure region over the airfoil upper surface and support the earlier result that dynamic lift is generated for compressible conditions.

8. Dynamic motion causes a lag in the pressure field development contributing to stall delay.

Further analysis of the leading edge flow images is underway.

\section{Acknowledgements}

The project was supported by an AFOSR grant (MIPR-92-0004) to the Naval Postgraduate School and was monitored by Maj. D.B. Fant. Additional support was received by ARO-MIPR-130-92, monitored by Dr. T.L. Doligalski. The work was carried out in the Fluid Mechanics Laboratory Branch of NASA Ames Research Center. The steady encouragement of Dr. S.S. Davis, Chief, FML Branch, the support of Mr. J.D. Loomis in the conduct of experiments and the interferogram processing software development effort of Mr. P.J. Trosin, Sterling Federal Systems, Inc., are greatly appreciated.

\section{References}

${ }^{1}$ Acharya, M. and Metwally, M.H., "Evolution of the Unsteady Pressure Field and Vorticity Production at the Surface of a Pitching Airfoil", AIAA Paper No. 90-1472, presented at the AIAA $21^{\text {st }}$ Fluid Dynamics, Plasma Dynamics and Lasers Conference, Seattle, WA, June $18-20,1990$.

${ }^{2}$ Francis, M.S. and Keese, J.E., "Airfoil Dynamic Stall Performance with Large-Amplitude Motions", AIAA Journal, Vol. 23, No. 11, Nov. 1985, pp. 1653-1659.

${ }^{3}$ Aihara, Y., Koyama, H., and Murashige, A., "Transient Aerodynamic Characteristics of a TwoDimensional Airfoil During Stepwise Incidence Variation", Journal of Aircraft, Vol. 2, No. 8, Aug. 1985, pp. 661-668.

${ }^{4}$ Albertson, J.A., Troutt, T.R., and Kedzie, C.R., "Unsteady Aerodynamic Forces at Low Airfoil Pitching Rates", AIAA Paper No. 88-2579-CP, Proc. $1^{\text {st }}$ National Fluid Dynamics Conference, Vol. 1, pp. 454-462, July 1988.

${ }^{5}$ Walker, J.M., Helin, H.E., and Strickland, J.H., "An Experimental Investigation of an Airfoil Undergoing Large-Amplitude Pitching Motions", $A I A A$ Journal, Vol. 23, No. 8, Aug. 1985, pp. 1141-1142.

${ }^{6}$ Jumper, E.J., Shreck, S.J., and Dimmick, R.L., "Lift-Curve Characteristics for an Airfoil Pitching at Constant Rate", Journal of Aircraft, Vol. 24, No. 10 , Oct. 1987, pp. 680-687.

${ }^{7}$ Lorber, P.F., and Carta, F.O., "Airfoil Dynamic Stall at Constant pitch Rate and High Reynolds Number", Journal of Aircraft, Vol. 25, No. 6. June 1988. pp. 548-556. 
${ }^{8}$ Visbal. M.R., "Dynamic Stall of a ConstantRate Pitching Airfoil". Journal of Aircraft, Vol. 27, No. 5, May 1990, pp. 400-407.

${ }^{9}$ Chandrasekhara, M.S., and Carr, L.W., "Flow Visualization Studies of the Mach Number Effects on the Dynamic Stall of an Oscillating Airfoil", Journal of Aircrafi, Vol. 27, No. 6, June 1990, pp. 516-522.

${ }^{10}$ Chandrasekhara, M.S., Carr, L.W., and Ahmed, S., "Comparison of Pitch rate History on Dynamic Stall", Proc. NASA/AFOSR/ARO Workshop on Physics of Forced Unsteady Separation, NASA-CP3144, pp. 23-28, April 17 - 19, 1990.

${ }^{1}$ Carr, L.W., and Chandrasekhara, M.S., "Design and Development of a Compressible Dynamic Stall Facility", Journal of Aircrafi, Vol. 29, No. 3, May-June 1992, pp. 314-318.

${ }^{12}$ Chandrasekhara, M.S., and Carr, L.W., "Design and Development of a Facility for Compressible Dynamic Stall Studies of a Rapidly Pitching Airfoil", ICIASF'89 RECORD, IEEE Publication 89CH27623, Sep. 1989, pp. 29-37.

${ }^{13}$ Carr, L.W., Chandrasekhara, M.S., Ahmed, S., and Brock, N.J., "A Study of Dynamic Stall Using Real Time Irterferometry", AlAA Paper No. 910007 , presented at the AIAA $29^{\text {th }}$ Aerospace Sciences Meeting, Reno, NV, January $7-11,1991$.

${ }^{14}$ Brock, N.J., Chandrasekhara, M.S., and Carr, L.W., "A Rtal Time Interferometry System for Unsteady Flow Measurements", ICIASF'91 RECORD, IEEE Publication 91CH3028-8, Oct. 1991, pp. 423430.

${ }^{15}$ Goldstein, R.J., "Fluid Mechanics Measurements", Hemisphere Publications, 1985.

${ }^{16}$ Chandrasekhara, M.S., Ahmed, S., and Carr, L.W., "Schlieren Studies of Compressibility Effects on Dynamic Stall of Airfoils in Transient Pitching Motion", To appear in Journal of Aircraft.

${ }^{17}$ Carr, L.W., Chandrasekhara, M.S., and Brock, N.J., "A Quantitative Study of Unsteady Compressible Flow Over an Oscillating Airfoil", AIAA Paper No. 91-1683, presented at the AIAA $22^{\text {nd }}$ Fluid Dynamics, Plasma Dynamics and Lasers Conference, Honolulu, HI, June 24-27, 1991. 


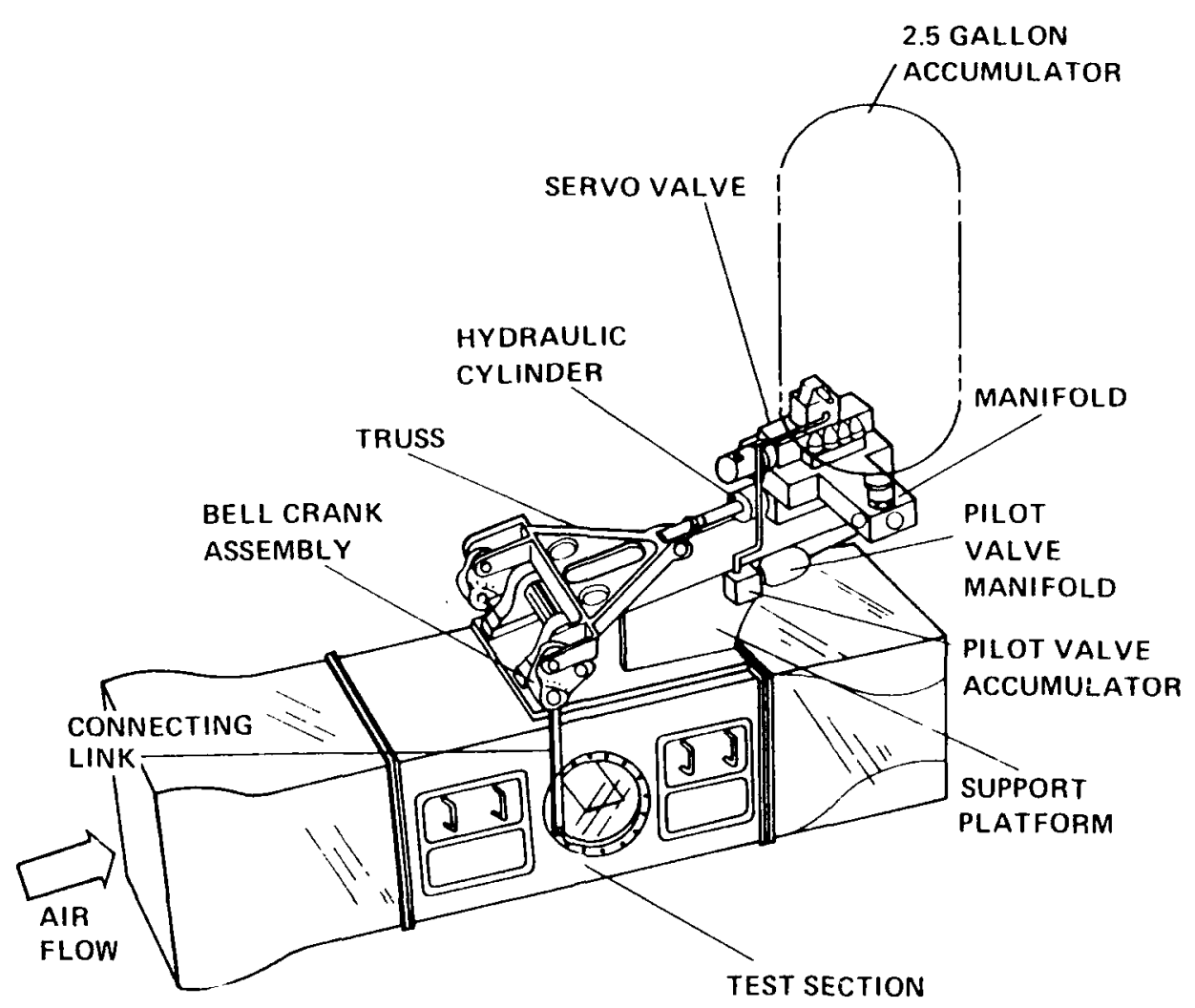

Fig. 1. Compressible Dynamic Stall Facility with a Hydraulic Drive.

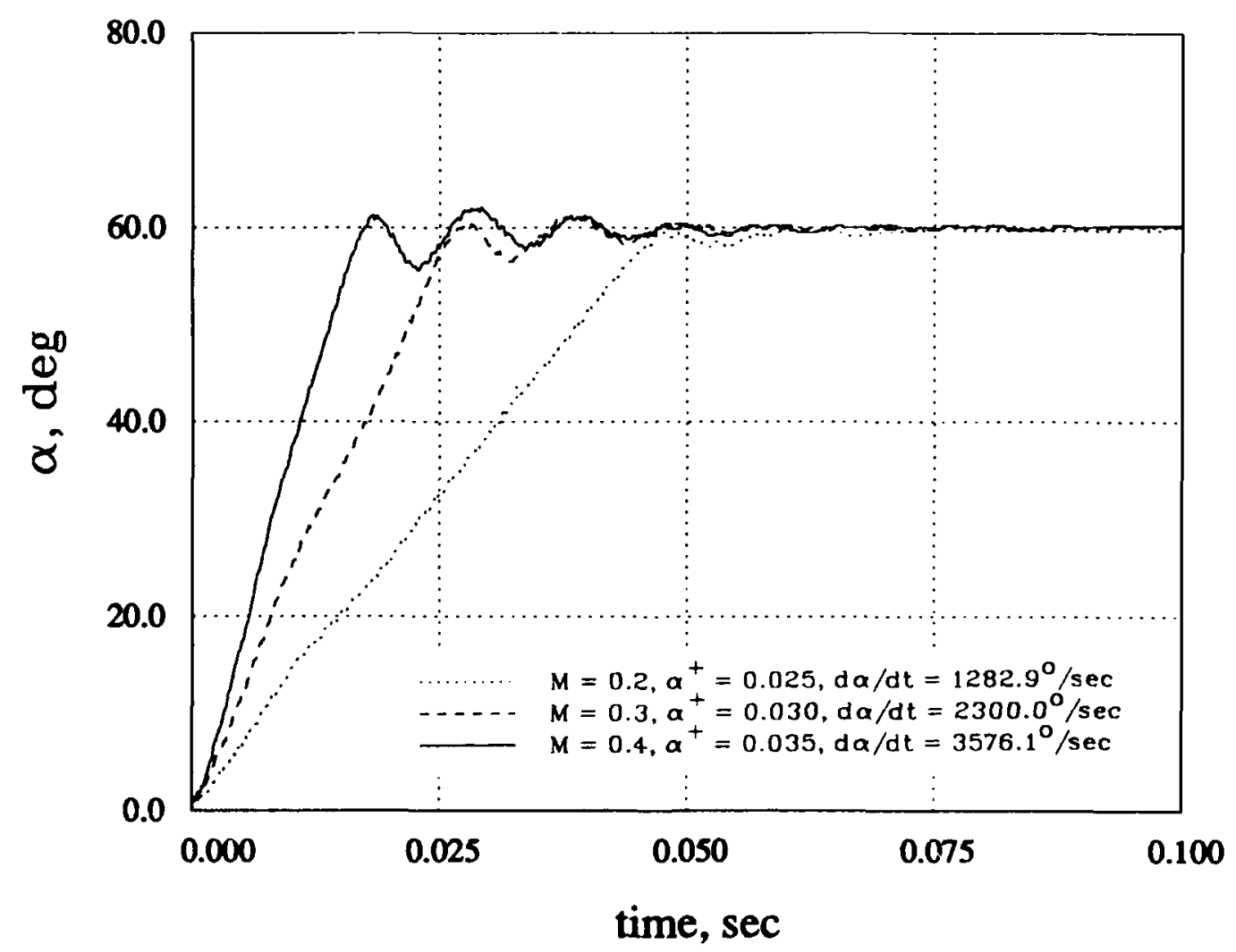

Fig. 2. Time History of Pitching Airfoil. 


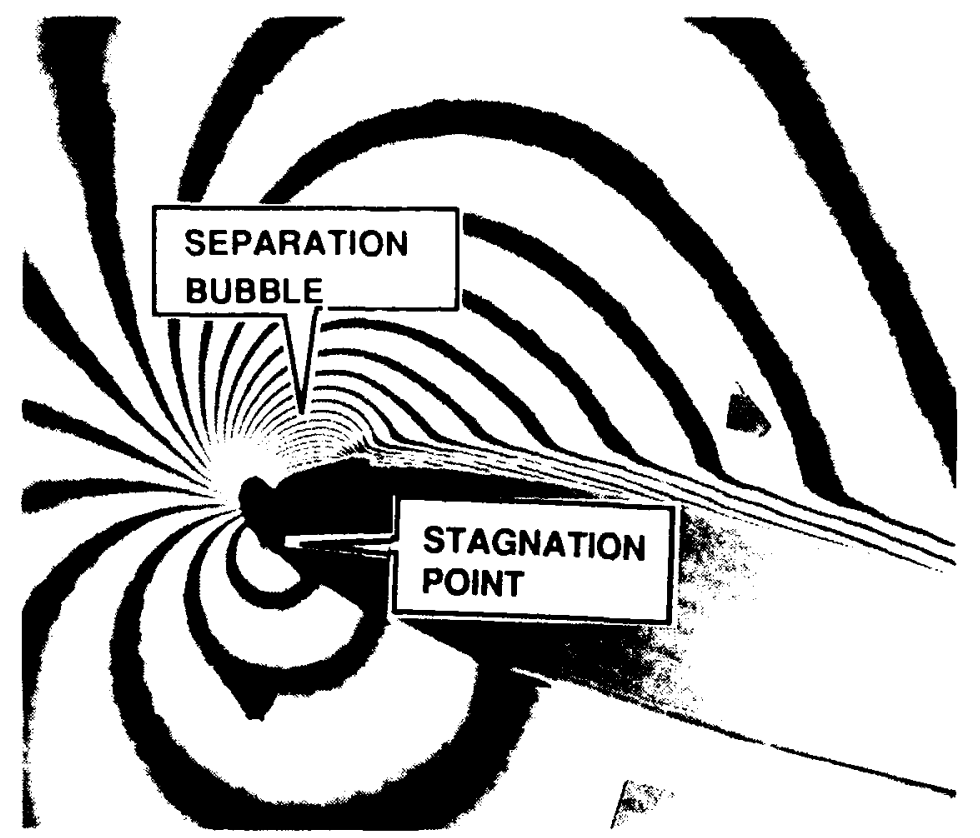

Fig. 3. Point Diffraction Interferogram at $M=0.3, \alpha=12^{0}, \alpha^{+}=0.03$.

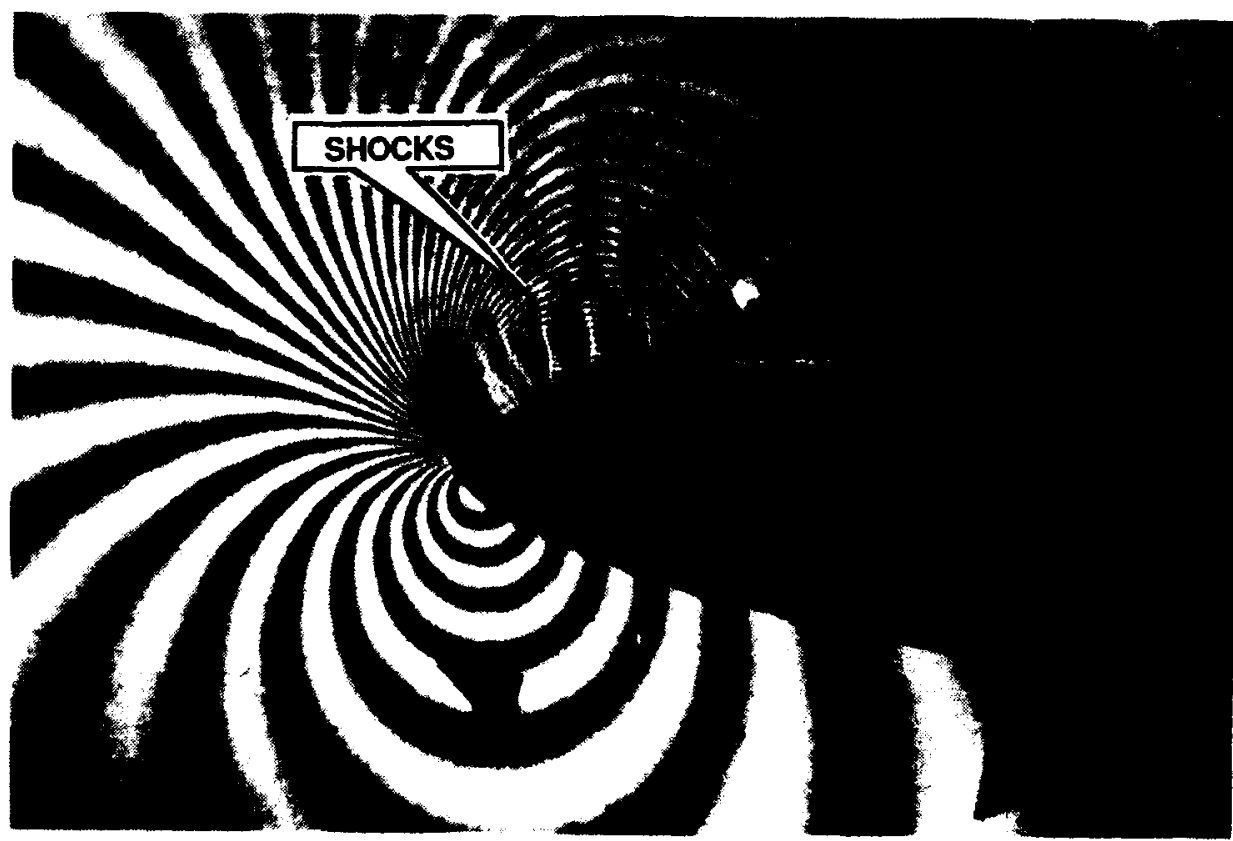

Fig. 4. Multiple Shocks over a Rapidly Pitching Airfoil, $M=0.45, \alpha=12.6^{0}, \alpha^{+}=0.0313$. 


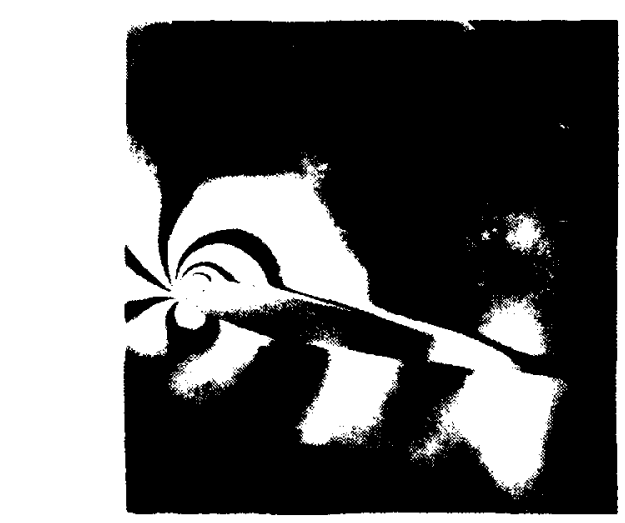

$\alpha=$

$12.0^{\circ}$

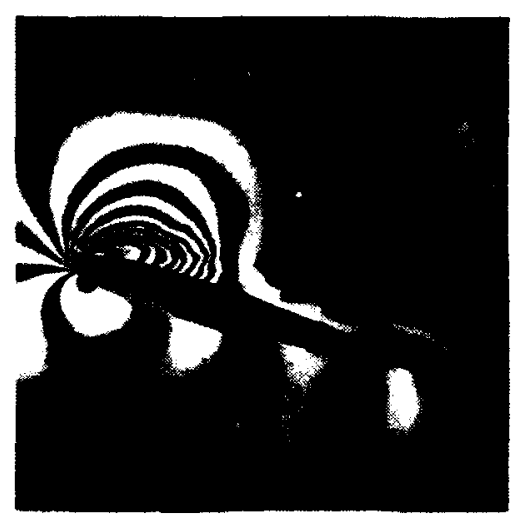

$$
\alpha=\quad 18.0^{\circ}
$$

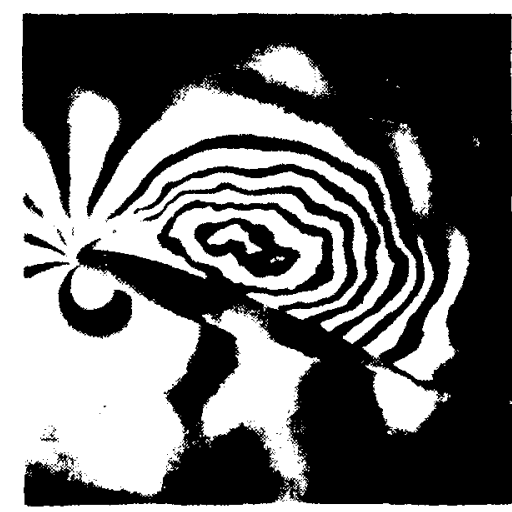

$\alpha=$

22.0

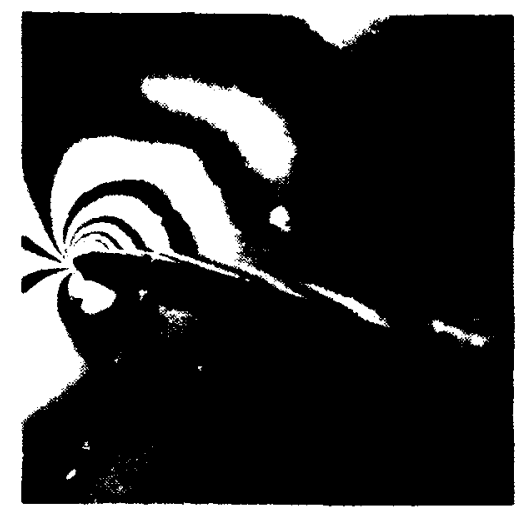

15.0

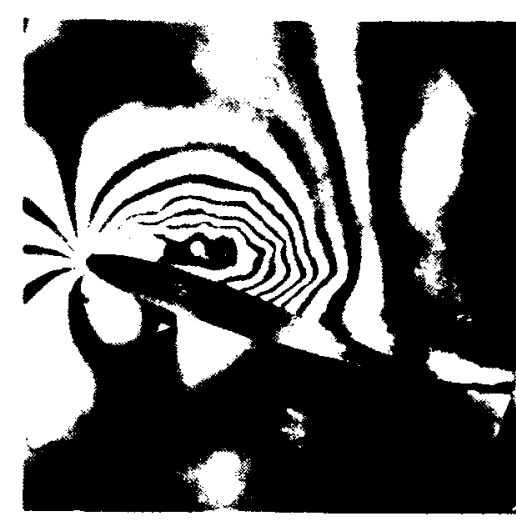

20.0

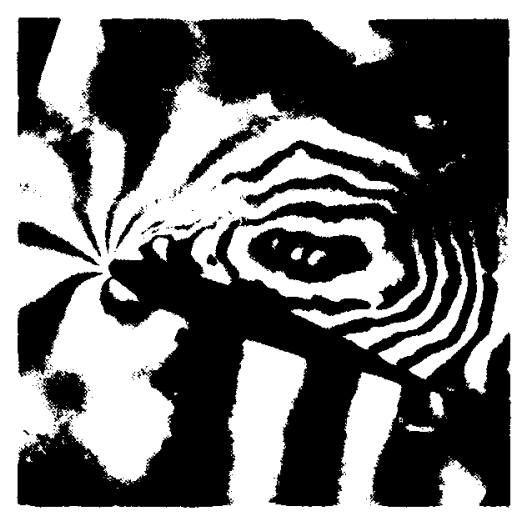

22.5

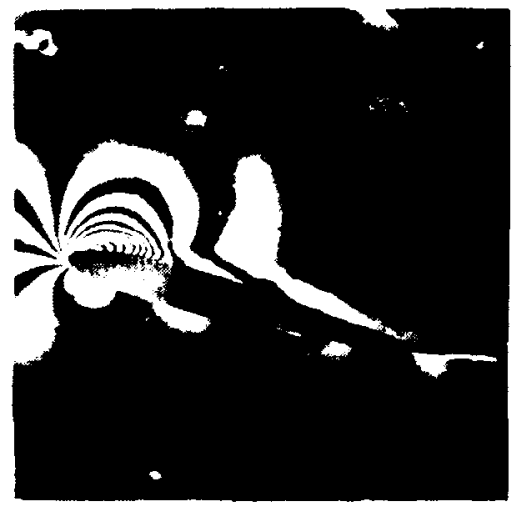

17.0

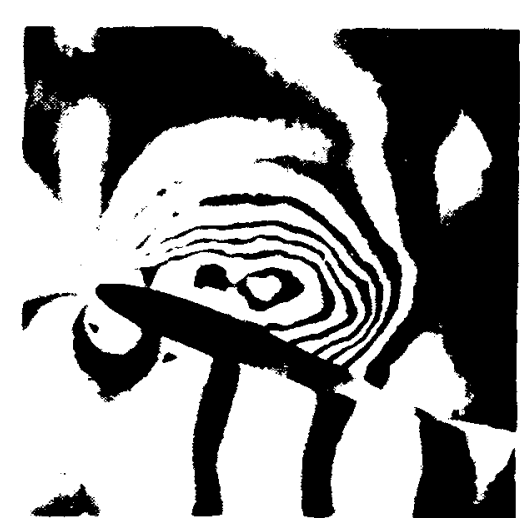

21.0

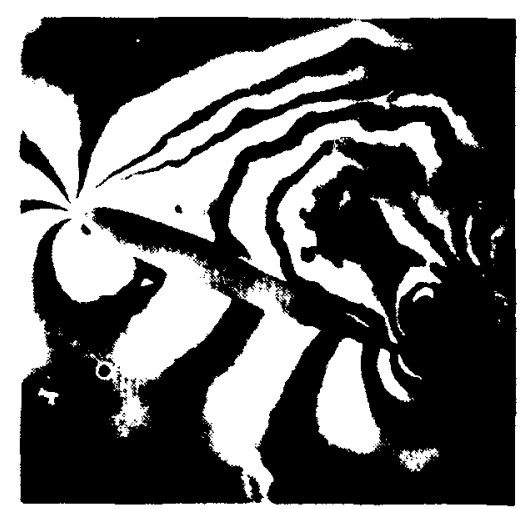

24.75

Fig. 5. Dynamic Stall Flow Development over a Transien:ly Pitching Airfoil, $M=0.2, \alpha^{+}=0.03$. 


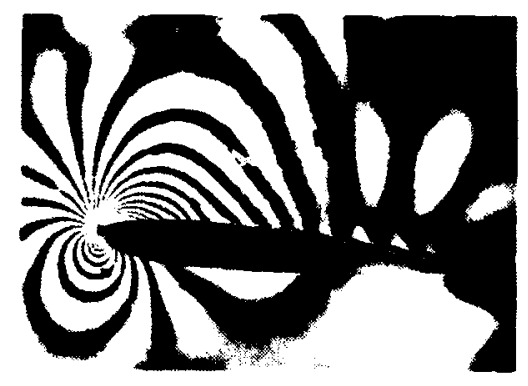

$x=$

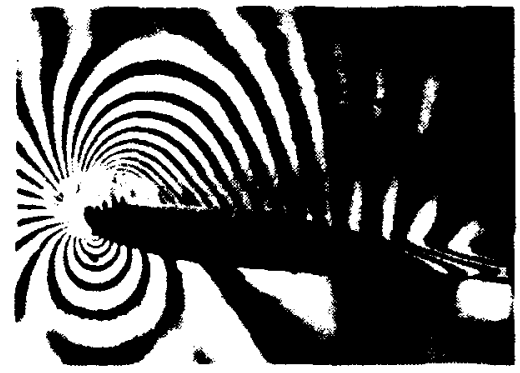

$\alpha=$

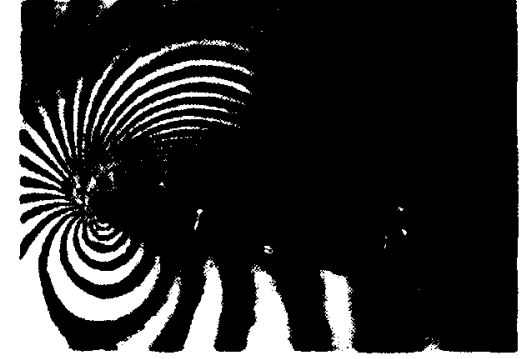

$\alpha=$ $13.0^{\circ}$

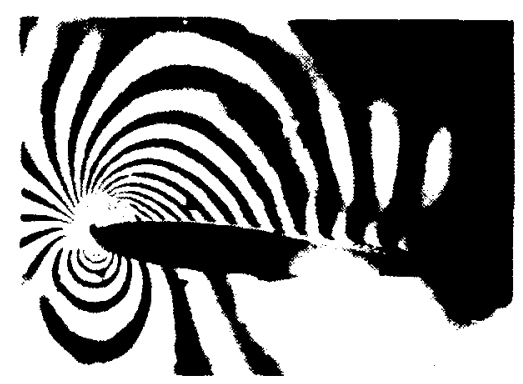

$7.0^{\circ}$

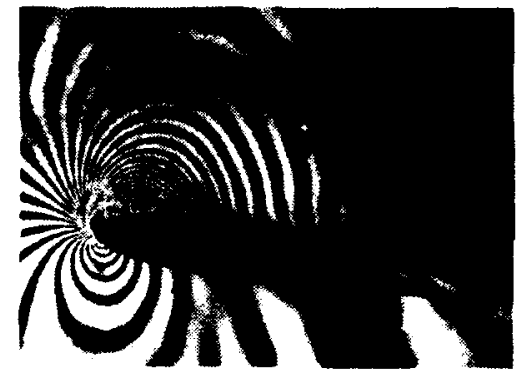

$11.0^{\circ}$

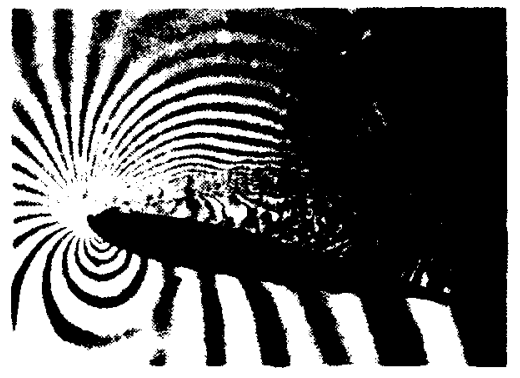

$14.0^{\circ}$

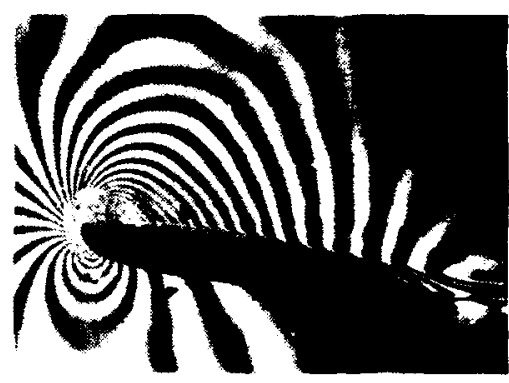

$9.0^{\circ}$

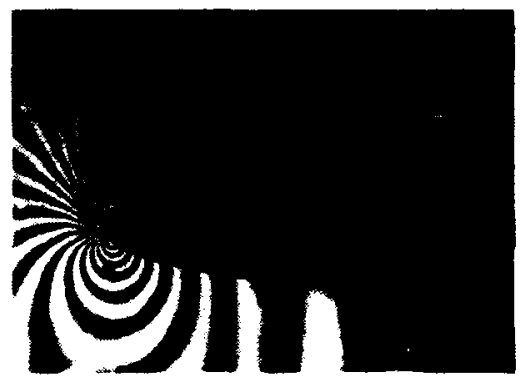

$12.0^{\circ}$

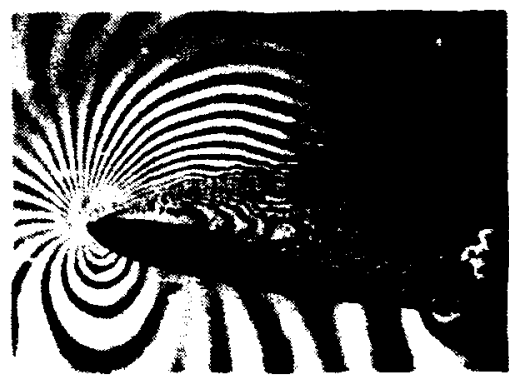

$14.5^{\circ}$

Fig. 6. Dynamic Stall Flow Development over a Transiently Pitching Airfoil, $M=0.45, \alpha^{+}=0.02$. 

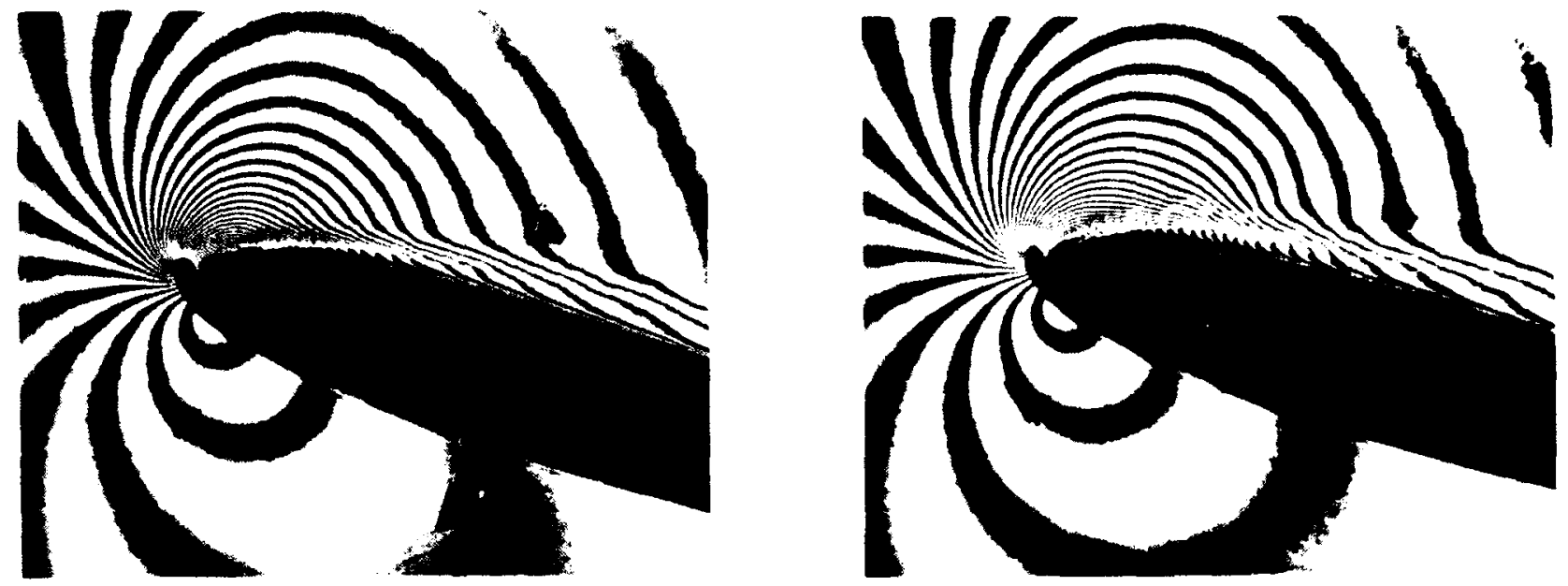

$\alpha=$

15.0

15.5
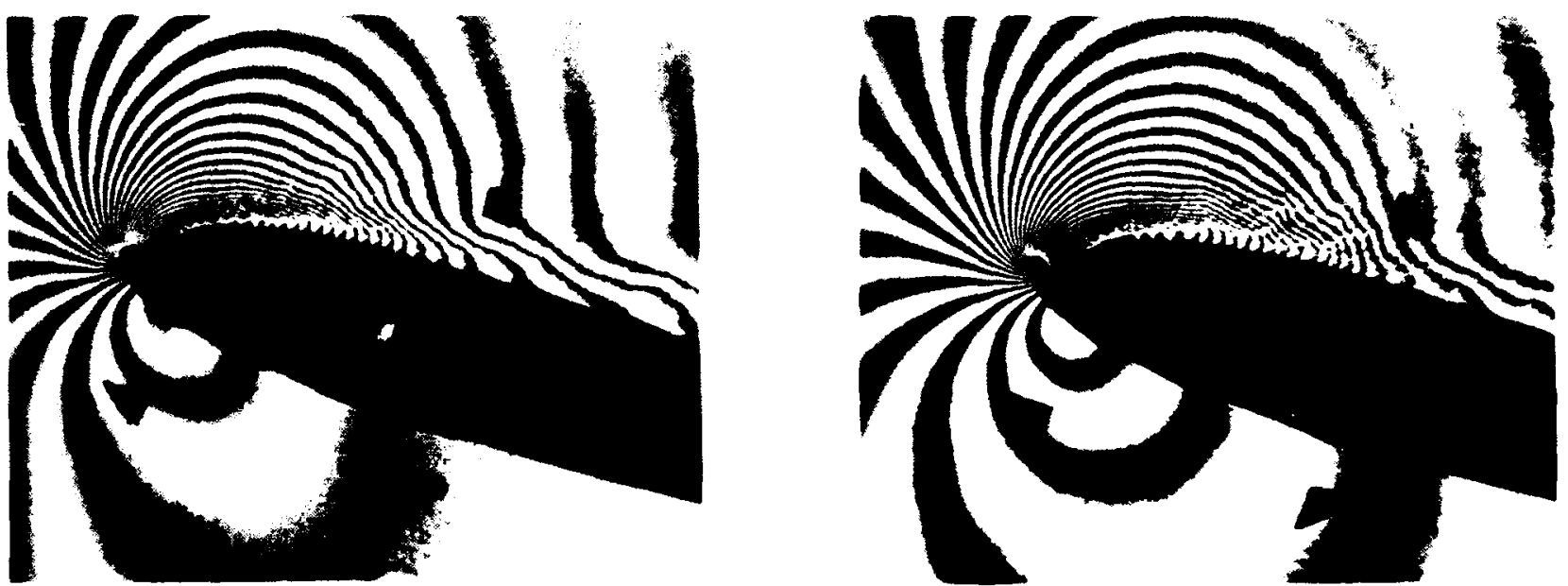

$\alpha=$

15.75

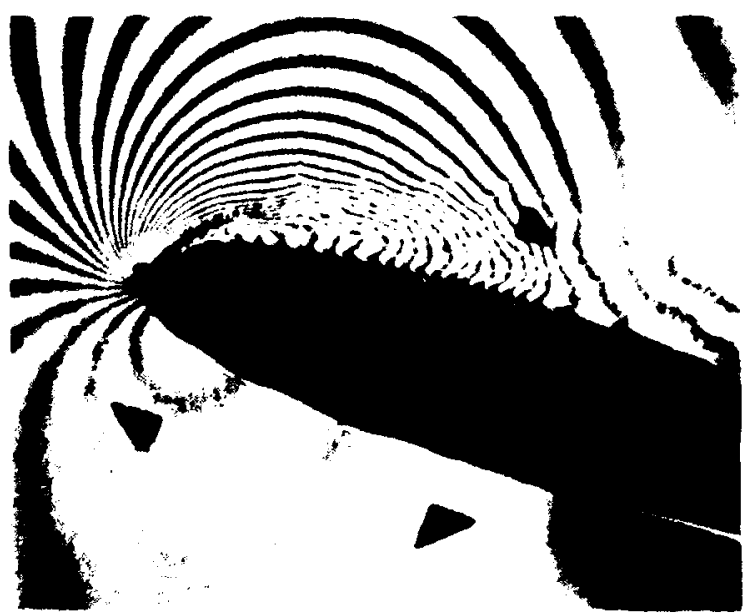

$\alpha=$

16.25

16.0

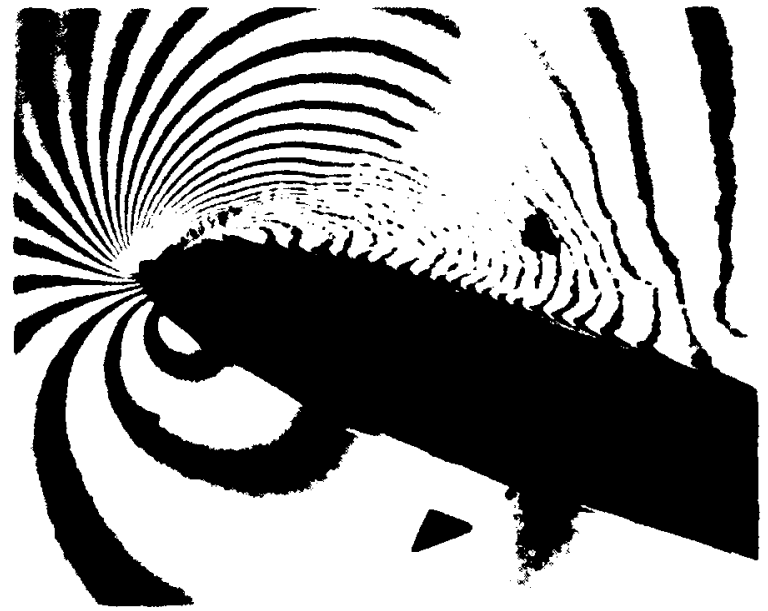

16.5

Fig. 7. Details of Dynamic Stall Vortex Development over a Transiently Pitching Airfoil, $M=0.3, \alpha^{+}=0.03$. 

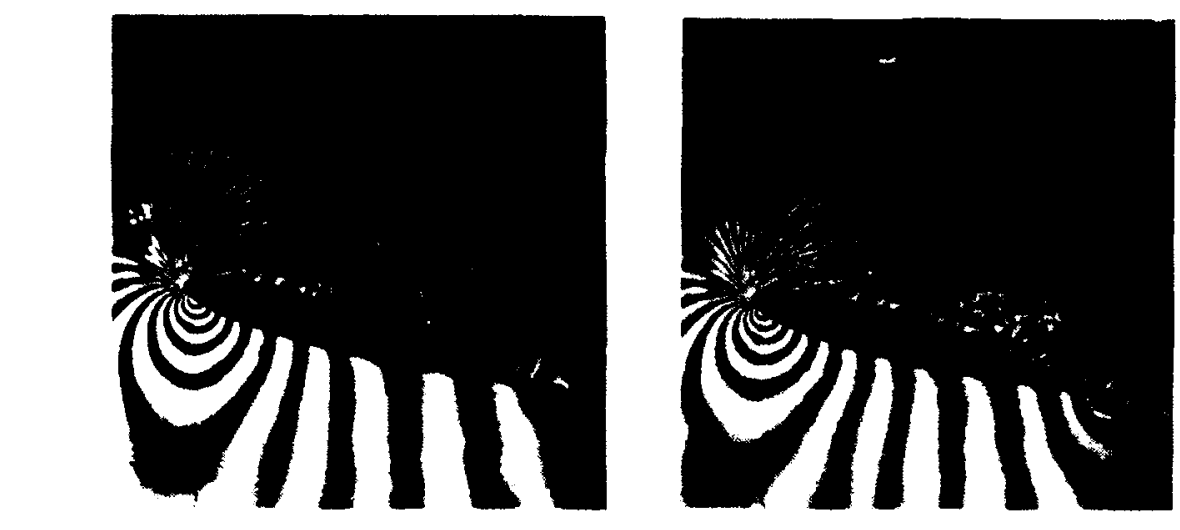

$\alpha=\quad 15.5^{\prime}$

16.0

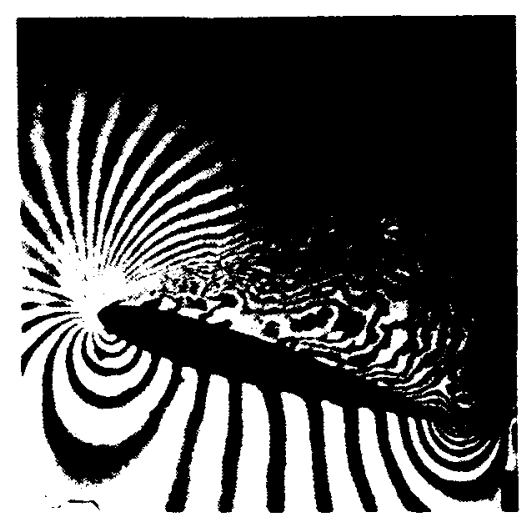

17.0

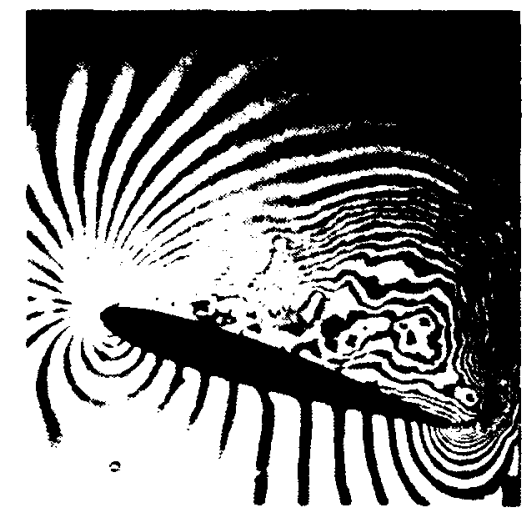

$\alpha=\quad 17.5$

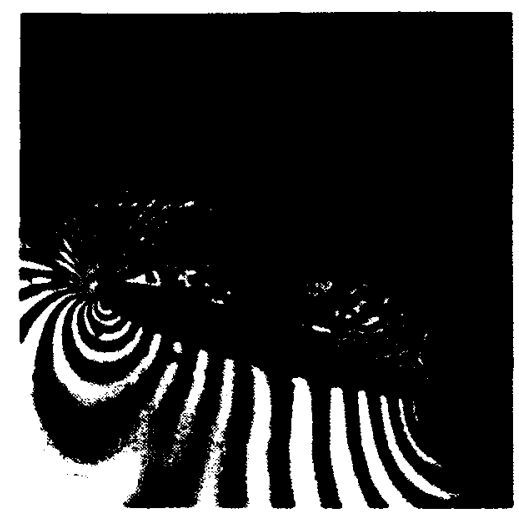

18.0

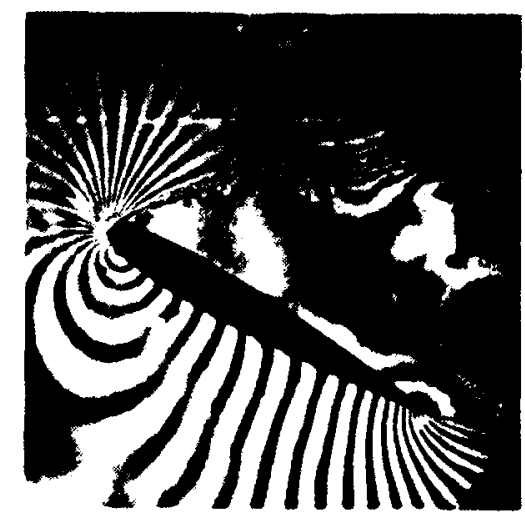

$\alpha=$ 30.0

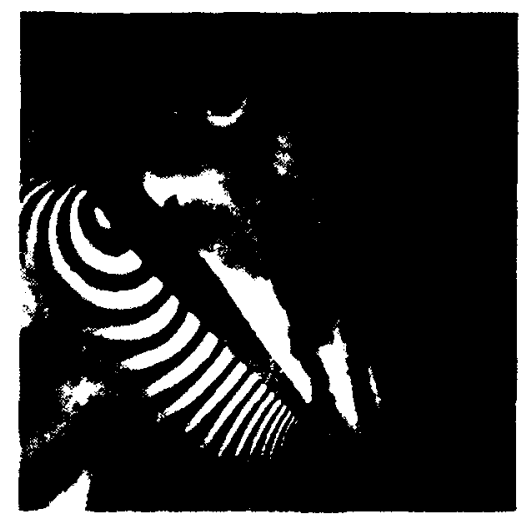

50.0

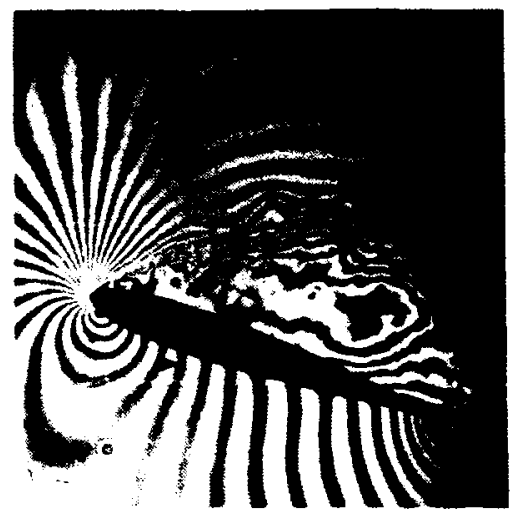

$20.0^{\circ}$

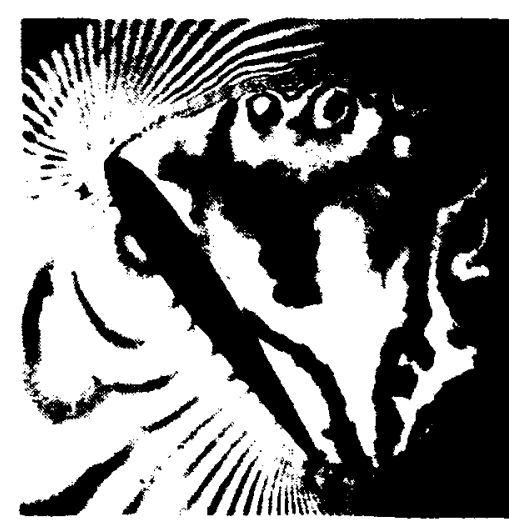

55.0

Fig. 8. Trailing Edge FLow Interactions with Dynamic Stall Vortical Flow over a Transiently Pitching Airfoil, $M=0.45, a^{+}=(0.025$. 


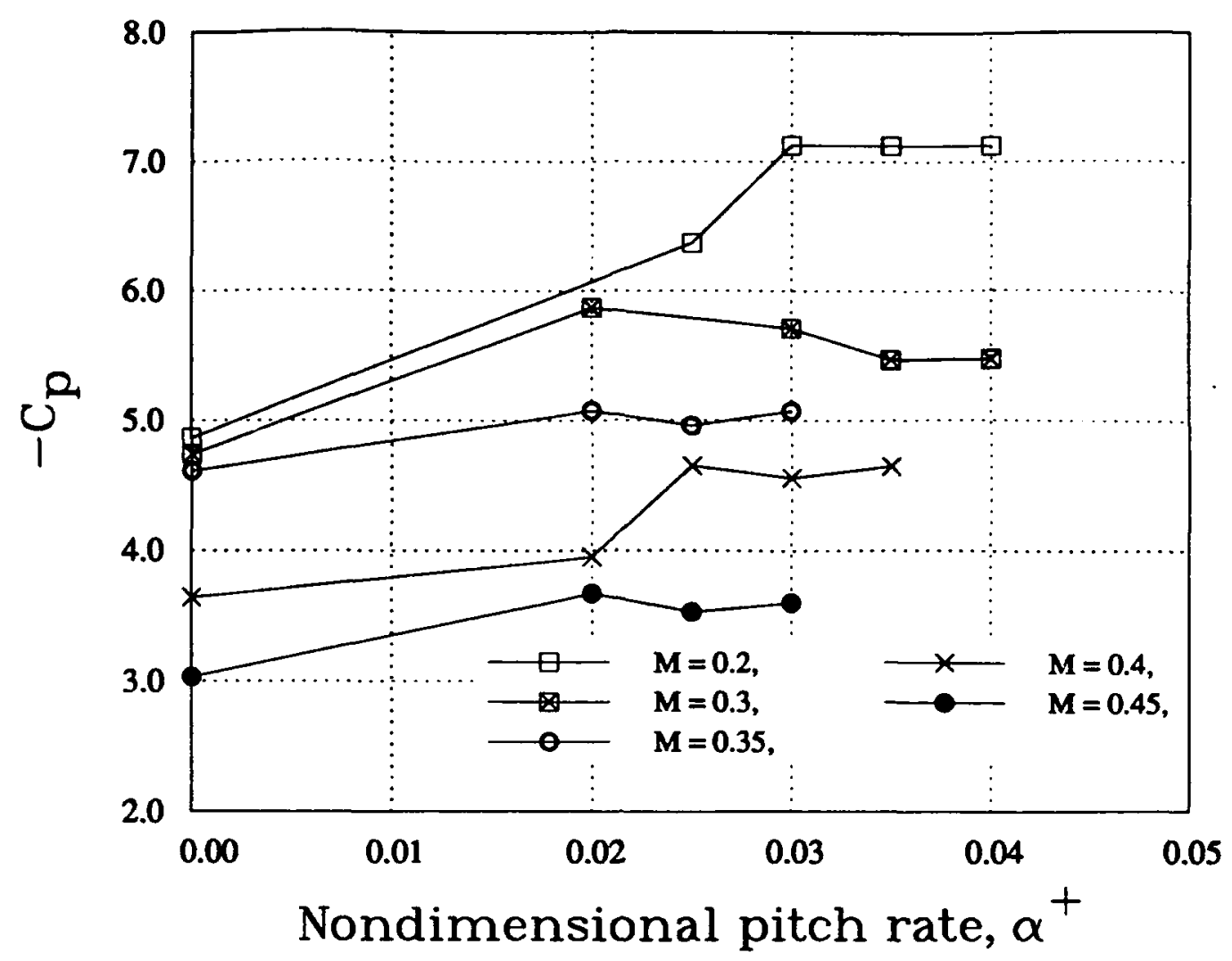

Fig. 9. Transiently Pitching Airfoil Peak Suction Pressure Coefficient at Stall Vortex Formation.

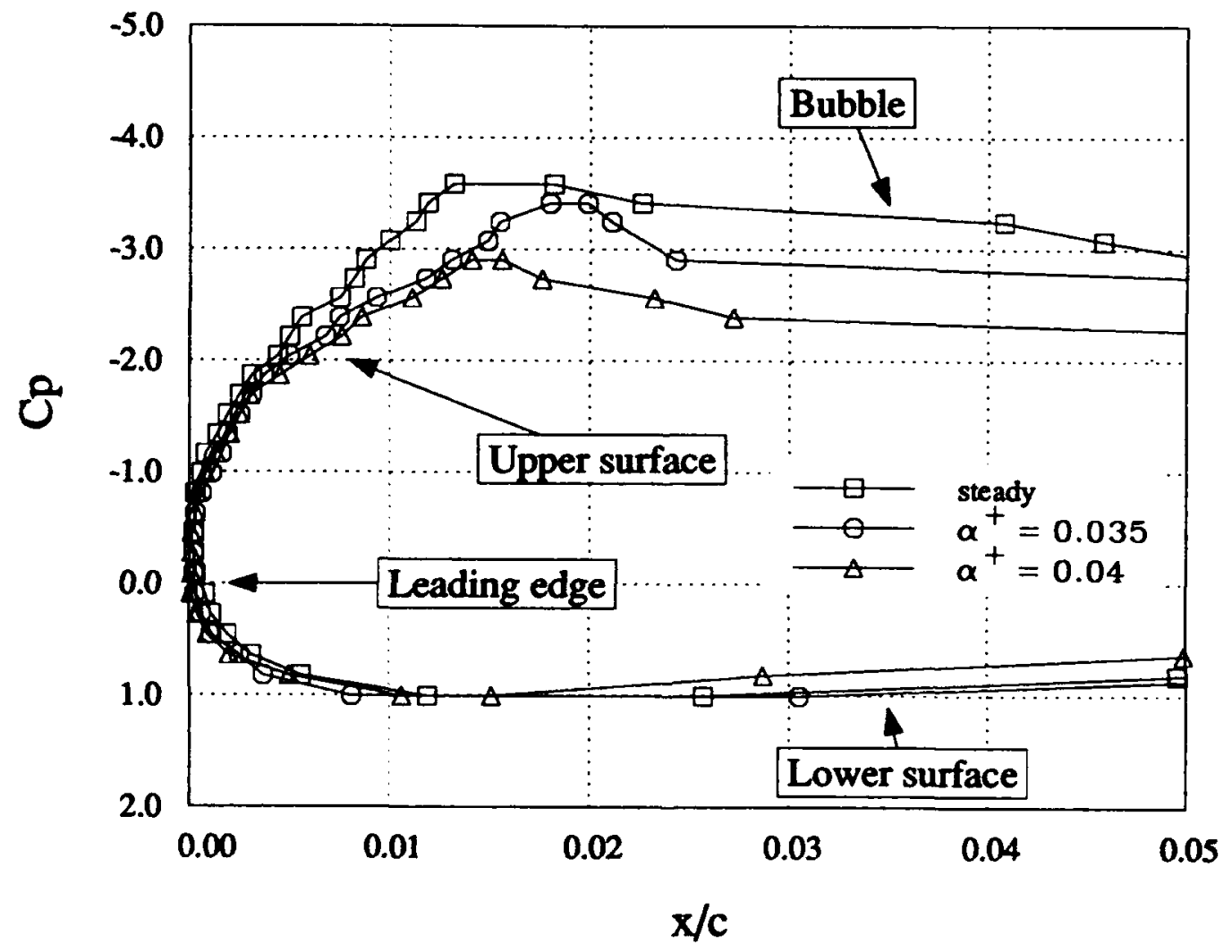

Fig. 10. Effect of Pitch Rate on Pressure Development over a Transiently Pitching Airfoil, $\mathbf{M}=0.3$. 


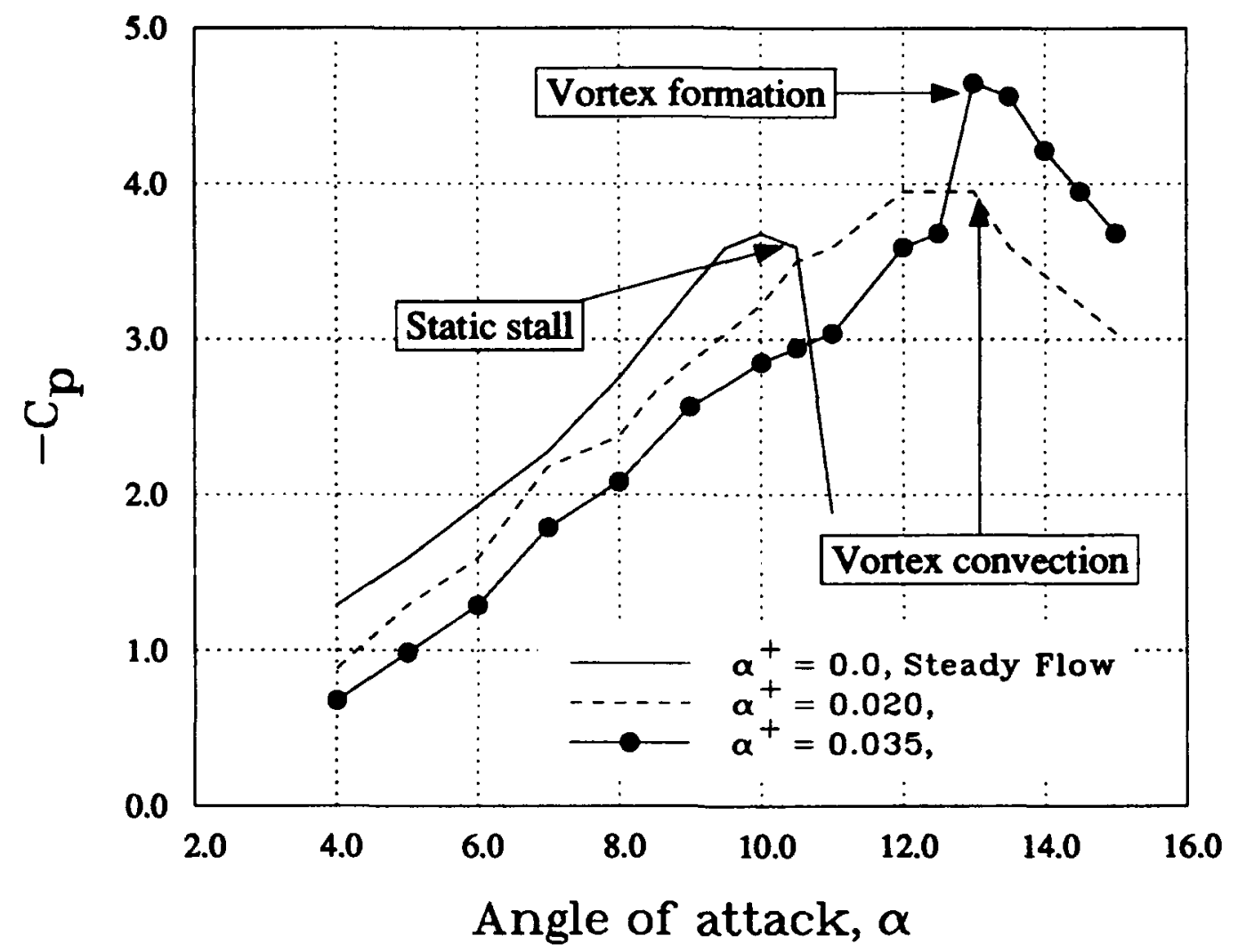

Fig. 11. Effect of Pitch Rate on Peak Suction Pressure Coefficient over a Transiently Pitching Airfoil, $\mathrm{M}=0.4$.

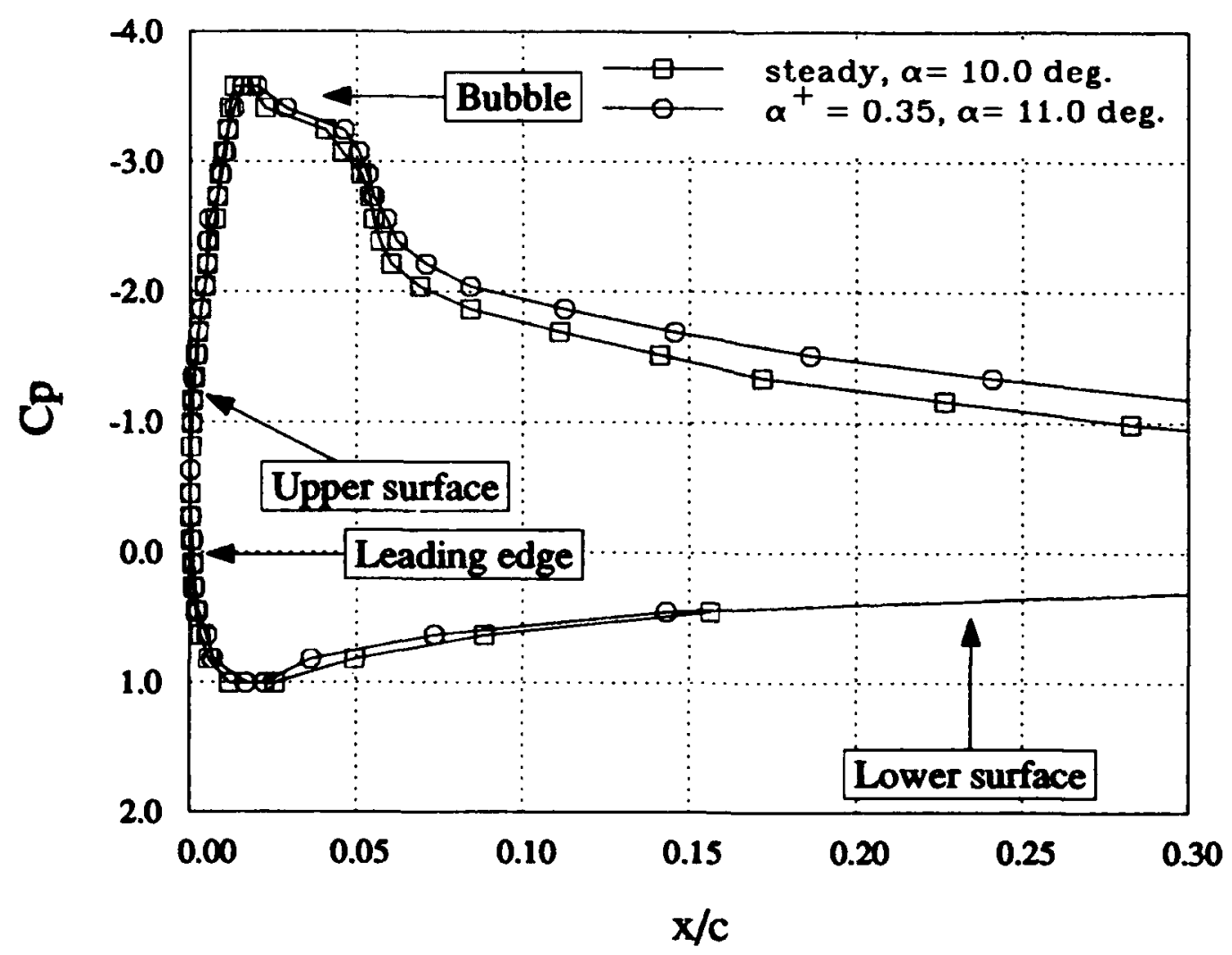

Fig. 12. Comparison of Steady and Unsteady Flows, $M=0.3$. 


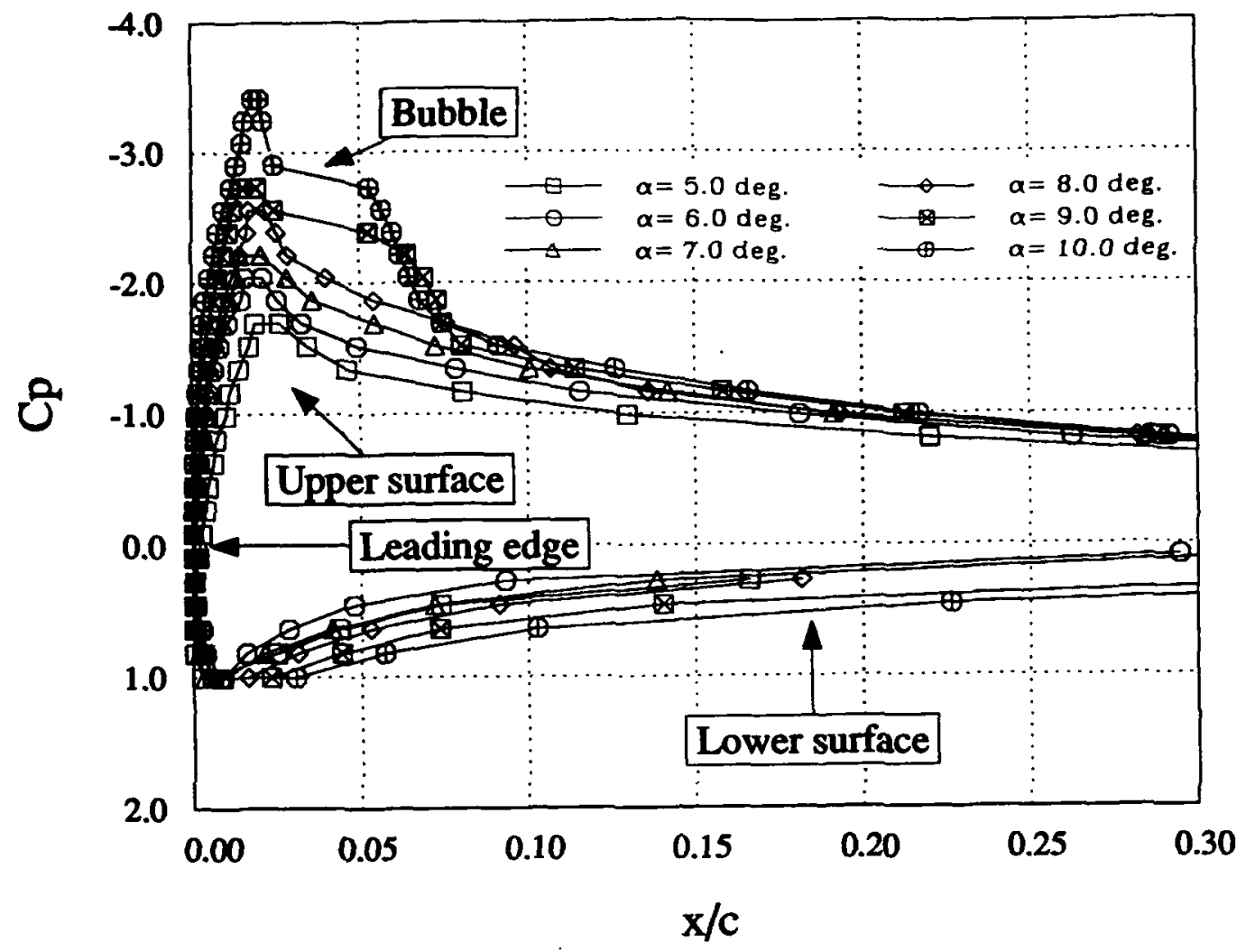

(a)

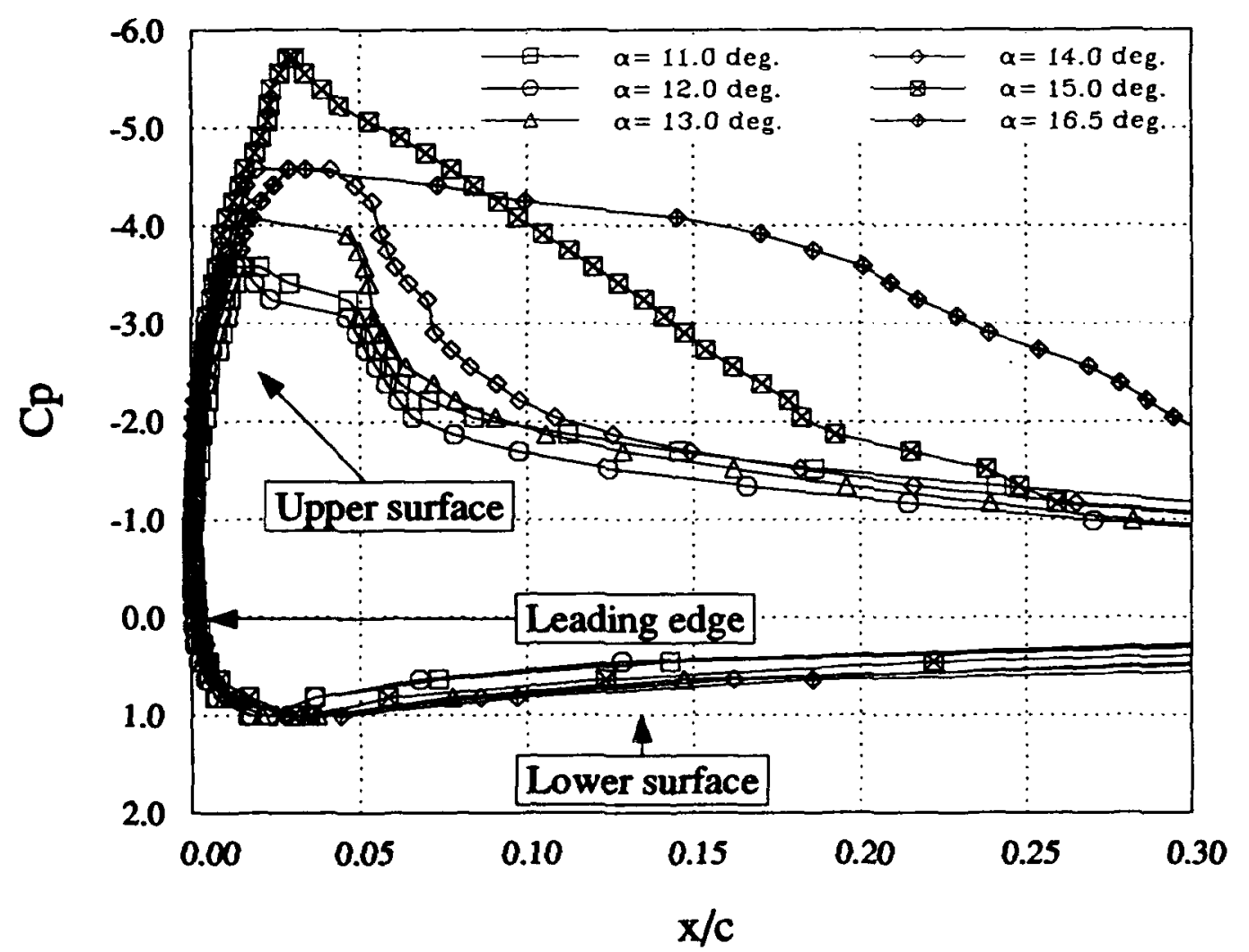

(b)

Fig. 13. Pressure Distribution over a Transiently Pitching Airfoil, $M=0.3, \alpha^{+}=0.35$. 